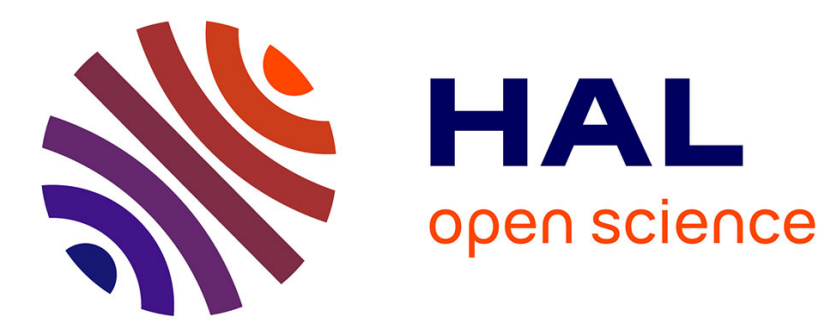

\title{
Probing the shear-band formation in granular media with sound waves
}

\author{
Yacine Khidas, X. Jia
}

\section{To cite this version:}

Yacine Khidas, X. Jia. Probing the shear-band formation in granular media with sound waves. Physical Review E : Statistical, Nonlinear, and Soft Matter Physics, 2012, 85 (5), pp.051302. 10.1103/PhysRevE.85.051302 . hal-00786223

\section{HAL Id: hal-00786223 \\ https://hal.science/hal-00786223}

Submitted on 31 Aug 2015

HAL is a multi-disciplinary open access archive for the deposit and dissemination of scientific research documents, whether they are published or not. The documents may come from teaching and research institutions in France or abroad, or from public or private research centers.
L'archive ouverte pluridisciplinaire HAL, est destinée au dépôt et à la diffusion de documents scientifiques de niveau recherche, publiés ou non, émanant des établissements d'enseignement et de recherche français ou étrangers, des laboratoires publics ou privés. 


\title{
Probing the shear-band formation in granular media with sound waves
}

\author{
Y. Khidas* and X. Jia ${ }^{\dagger}$ \\ Université Paris-Est, Laboratoire de Physique des Matériaux divisés et Interfaces, CNRS FRE 3300, 5 Bd. Descartes, \\ 77454 Marne-la-Vallée cedex 2, France
}

(Received 30 January 2012; published 3 May 2012)

\begin{abstract}
We investigate the mechanical responses of dense granular materials, using a direct shear box combined with simultaneous acoustic measurements. Measured shear wave speeds evidence the structural change of the material under shear, from the jammed state to the flowing state. There is a clear acoustic signature when the shear band is formed. Subjected to cyclic shear, both shear stress and wave speed show the strong hysteretic dependence on the shear strain, likely associated with the geometry change in the packing structure. Moreover, the correlation function of configuration-specific multiply scattered waves reveals an intermittent behavior before the failure of material.
\end{abstract}

DOI: 10.1103/PhysRevE.85.051302

PACS number(s): 45.70.-n, 83.80.Fg, 91.30.-f, 43.35.+d

\section{INTRODUCTION}

Granular materials are ubiquitous in everyday life, ranging from industrial applications to geophysical processes (e.g., fault core dynamics). If the steady-state granular flow is reasonably well understood $[1,2]$, the transition from the jammed state to the flowing state still remains elusive, including stick-slip behavior and precursor events before failure [3-6].

Many works on numerical simulations in two-dimensional (2D) or three-dimensional (3D) granular media have been performed to examine the evolution of the structure and the mechanical properties of a granular medium at micro- and mesoscales during the jamming transition and/or shear-band formation [7-9]. Meanwhile, only a few experiments are available, via photoelastic visualization [10] in 2D and x-ray $[11,12]$ and magnetic resonance imaging (MRI) tomography in 3D granular models [1], to investigate their microstructure changes and the particle velocity fields during slow flow. However, the application of these experimental methods to real 3D opaque granular materials appears difficult.

Sound waves provide a unique and sensitive probe for investigating the contact force networks in real granular media [13-17]. Speed measurements of long-wavelength sound waves allow one to determine the nonlinear elasticity and anisotropic effects of the granular material [14-16], while short-wavelength multiply scattered sound waves enable one to detect tiny changes of the contact network configuration at the grain level [17]. Sound waves also provide useful information about the nonlinear dynamics of granular materials under slow shear flow [18] and during granular avalanche [19]. However, is there any specific acoustic signature of the shear-band formation obtainable via shear sound reflection or transmission [20]? Probing with the shear wave might allow inferring the specific behavior of the shear modulus near unjamming transition [21].

In this work, we show that the sound speed measurements in transmission and particularly of the shear wave provide an

\footnotetext{
*Present address: Université Paris-Est, Laboratoire Navier, 77454 Marne-la-Vallée cedex 2.

†jia@univ-mlv.fr
}

assessment of changes in the granular structure of a glass bead packing under shear loading. Our data reveal a clear acoustic signature when the shear band is formed inside the medium. Special attention will be paid to the hysteretic behavior of the mechanical responses and the shear wave speed evolutions under cyclic shear loading with ramped strain amplitude. We will also investigate the irreversible rearrangements of the contact networks under shear by multiply scattered sound waves in both dense and loose granular packings.

\section{APPARATUS}

To create the localization zones of shear strain away from the wall boundaries, we utilize here a direct shear apparatus shown in Fig. 1(a). It consists of a metal box of square cross section $40 \times 40 \mathrm{~mm}^{2}$ separated into two parts and each has a height of $15 \mathrm{~mm}$ (with a filling height $H=30 \mathrm{~mm}$ ). The shear loading is applied horizontally to the lower part of the box at the constant velocity $V_{\text {drive }}=0.6 \mu \mathrm{m} / \mathrm{s}$, while the upper part is kept fixed. The shear force $T$ required to maintain the upper part immobile is measured by a load cell of stiffness $k=10^{7} \mathrm{~N} / \mathrm{m}$. The constant normal load $N=330 \mathrm{~N}$ is applied to a piston on the top of the granular sample, corresponding to a normal stress of $P=206 \mathrm{kPa}$. A position sensor records the relative horizontal displacement between the two parts of the shear box. A second one measures the vertical displacement of the piston to determine the dilatancy of the material induced by shear.

Spherical glass beads of diameter $d=700 \pm 20 \mu \mathrm{m}$ are used. The material constants of the glass beads are $\rho=$ $2500 \mathrm{~kg} / \mathrm{m}$ for the bulk density, $G=25 \mathrm{GPa}$ for the shear modulus, and $v=0.2$ for the Poisson ratio. To investigate the influence of the packing density on the unjamming transition in sheared granular materials, two different packing protocols are performed. For the "rainfall" packing preparation, the beads are poured into the box through two spaced grids. This packing protocol creates the dense packing with a solid volume fraction of $\phi=0.641 \pm 0.002$. For the decompaction packing protocol, we slowly remove an inner horizontal grid through the packing. This preparation method gives a loose packing with $\phi=0.600 \pm 0.002$. Note that these two preparation methods also produce different granular fabrics [16]. 
(a)
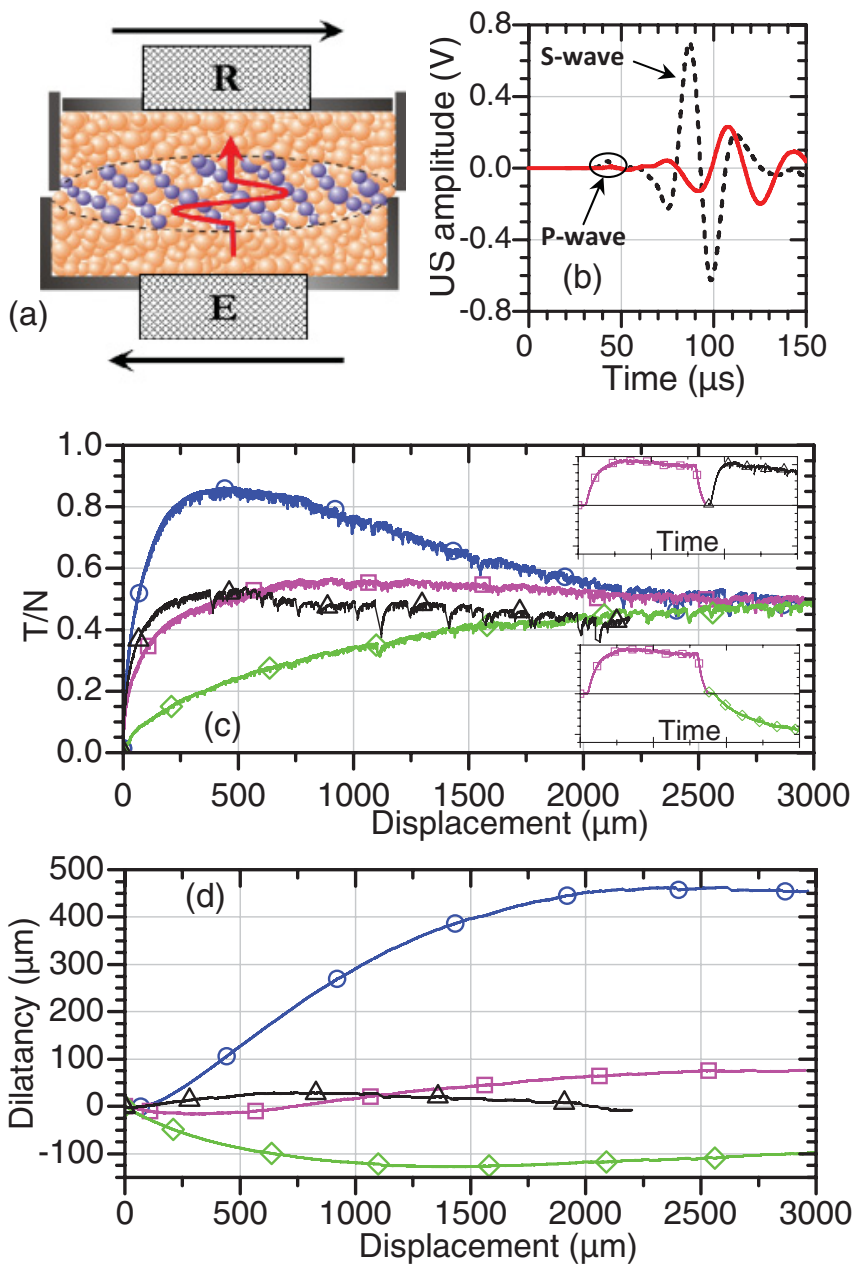

FIG. 1. (Color online) (a) Direct shear apparatus combined with acoustic measurements where $E$ and $R$ are the acoustic source and receiver. (b) Typical transmitted ultrasonic signals obtained before shear loading (dashed line) and at the peak shear force (solid line). (c) Evolution under shear of force ratio $T / N$ and (d) dilatancy in the loose $(\boxminus)$, dense $(\ominus)$, and stratified packings ( $\triangle$, reloading in the same direction; $\vartheta$, reloading in the reverse direction).

Combined ultrasonic measurements are conducted simultaneously with the shear experiments. One is the speed measurement of coherent shear waves and the other is the detection of multiply scattered waves. As shown in Fig. 1(a), a large shear transducer of diameter $30 \mathrm{~mm}$ is placed on the lower part of the shear box. For the speed measurement, this source transducer of broadband, centered at $250 \mathrm{kHz}$, is excited by a short pulse of $4 \mu \mathrm{s}$ and coherent shear pulses with central frequencies around $40 \mathrm{kHz}$ are measured by the same large transducer placed in the upper piston at various shear loading [Fig. 1(b)]. The low-frequency spectra of these coherent waves are basically due to scattering attenuation [14]; no dispersion of sound speed is observed here. To avoid the interference between the compressional signals and the dominant shear pulses caused by the small size of the shear box [16], we measure the shear wave speed $V_{S}$ via the time of flight of the pulses maxima (the real value of $V_{S}$ is thus underestimated). The relative change of the shear wave speed $\Delta V_{S} / V_{S}$ due to shearing shown in Fig. 1(b) is about 22\%, 24\%, or $27 \%$ if one follows the evolution of the maxima, first, or second minima, respectively; these results are consistent showing thus fairly accurate measurements of $\Delta V_{S} / V_{S}$. For monitoring the local rearrangement by the acoustic speckles, the multiply scattered waves are generated by a ten-cycle burst centered at $250 \mathrm{KHz}$ and are detected by a small transducer of $2 \mathrm{~mm}$ placed at the top.

\section{MECHANICAL RESPONSES}

We first examine the mechanical response of a granular medium under shear. Figures 1(c) and 1(d) show typical evolutions of the force ratio $T / N$ and the dilatancy as a function of the relative horizontal displacement $U$ between the two parts of the shear box. We observe the classical behavior of sheared granular media, undergoing the transition from the jammed state to the flowing state. For the densely packed sample, the shear force $T$ rises rapidly with the shear displacement in the early stage before reaching a peak value and then decreases and tends to a stationary value when the flow is fully developed. For the loosely packed sample, the behavior is similar except that there is no peak force. In both cases, there are small compressions of the media at the initial stage preceding the dilatations [Fig. 1(d)].

Note that the steady-state flowing is characterized by a constant value of $T / N$, referred to as the residual strength; it is independent of the initial sample density and the fabric anisotropy [22]. It has been shown that when a granular medium is sheared to the steady state in a direct shear box, the shear strain is essentially localized in a narrow zone located at the midheight of the box where a shear band is formed. Such a shear localization zone exhibits distinct features compared to the rest of the medium, including extremely large voids and the presence of a highly anisotropic network of force chains $[7,9,12]$.

We then wonder what may be the mechanical behavior of a granular medium comprising a preexisting shear band when applying a shear force. Figure 1(c) (insets) displays the shear force displacement response of such a sample, sheared either in the same or the reverse direction as the first shearing. In such stratified medium possessing a shear band at the middle, the peak force disappears and the medium reaches closely the same steady flowing state. When the reloading is performed in the same direction as the prior shear direction, we observe a similar mechanical behavior to that in a loosely packed sample. As mentioned previously, when all the samples are driven to the steady-state flow, the shear strain is localized in the shear band. The same stationary value of the ratio $T / N$ implies that a fully developed shear band has the same properties, independent of the sample history.

\section{ACOUSTIC RESPONSES}

We now examine the evolution of shear wave speed $V_{S}$ through the granular samples during the first shear loading before the strain localization. As shown in Fig. 2, significant decreases of shear wave speed $V_{S}$ up to $20 \%$ are observed in both densely and loosely packed samples, displaying a comparable overall acoustic response under shear. For large shear displacement, the steady-state flow is characterized by 

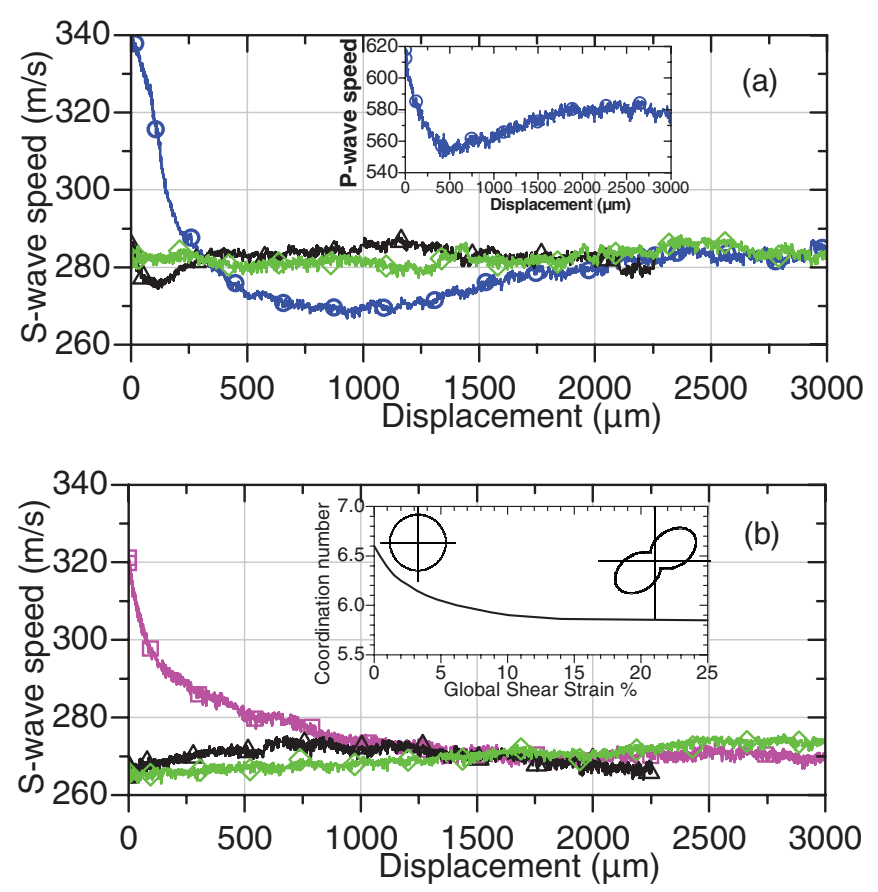

FIG. 2. (Color online) Evolutions of the shear wave speed under shear in the dense samples (a) and loose samples (b). $\boxminus$ and $\ominus$, first loading; $A$, reloading in the same direction; $\theta$, reloading in the reverse direction. Inset in (a): decrease of the compression wave speed during shear. Inset in (b): illustration of the evolution of the coordination number obtained in 3D discrete element model simulation in [8] with the polar distribution evolution of contact normal force inspired from [9].

a constant wave speed. As a comparison, we note that the decrease of the compression wave speed $V_{P}$ during shear [inset of Fig. 2(a)], measured with longitudinal transducers, is about two times less than that of $V_{S}$.

To account for our speed measurements during the transition from the jammed state to the flowing one, we may compare qualitatively the experiments with the predictions by the effective medium theory (EMT) [15,23]. In the limit of long wavelength as in our experiments, i.e., $\lambda \sim 10 \mathrm{~mm}$ much larger than the bead size $d \sim 0.7 \mathrm{~mm}$, the speeds of compression and shear waves $V_{P}$ and $V_{S}$ are related to the effective bulk and shear modulus $K$ and $G$ via $V_{P}=[(K+2 G / \rho)]^{1 / 2}$ and $V_{S}=$ $(G / \rho)^{1 / 2}$, where $\rho=\rho_{0} \phi$ and $\rho_{0}$ is the glass bead density. For isotropic compression $P$, the scaling expression of elastic moduli $K$ and $G$ yields [15]

$$
V_{P, S} \sim Z^{1 / 3} \phi^{-1 / 6} P^{1 / 6},
$$

with $Z$ the coordination number, i.e., the mean number of contact per grain. This simplified expression illustrates a general relationship between the microstructural parameters and the macroscopic acoustic properties. The overall speed decreases of compression and shear waves observed under shear is thus likely related to the change of the geometric structure (i.e., texture) with a decrease of the mean coordination number $Z$. The inset of Fig. 2(b) shows 3D numerical simulations of direct shear test [8] in which the decrease of the coordination number induced by shearing is clearly evidenced, supporting thus our interpretation.
Nevertheless, the anisotropy of the stress field induced by shearing may also affect our measured wave speeds. As shown previously [16], the scaling relationship in Eq. (1) holds qualitatively for anisotropic loading if $P$ corresponds to the stress component along the direction of wave propagation. Applying a shear load here induces the principal axis rotation of the stress and the modulus tensor, which reduces effectively the elastic moduli $K$ and $G$ and consequently, the sound speeds $V_{P}$ and $V_{S}$ along the vertical direction. This anisotropic effect could also explain the evolution of wave speed in the densely packed sample, which passes through a minimum before reaching the stationary value. Indeed, when the dense packing reaches the pronounced peak force [Fig. 1(c)], the principal stress direction is deviated from the vertical direction significantly reducing the sound speeds. When the medium attains the flowing state, the principal axis of stress rotates back to the vertical direction. This behavior has been confirmed by numerical simulation on the evolution of either the deviator fabric in 3D simulations [8] or the distribution of normal contact forces in 2D simulations [9].

The main finding of this work lies in the drastically different acoustic response in a granular medium where the shear band is fully developed, e.g., by the first loading. Under further shearing, whatever the direction of reloading versus that of the prior shearing, the shear wave speed in such a granular medium remains nearly constant (Fig. 2). This observation provides a clear acoustic signature of the irreversible modification inside the granular structure with the formation of a shear band. We understand this result as follows. During the first loading, a shear band is formed which becomes the weakest zone of the medium. Upon reloading, this weak zone localizes the strain and yields immediately, driving the medium to the flowing state. However, the structure away from this narrow shear band (a few grain size) in the rest of the sample, is little deformed; hence, the coordination number and the anisotropy of the medium remains globally unchanged. The different stationary values of $V_{S}$ between the dense and loose packings reveal a dependence on the initial packing condition. Note that there is no detectable acoustic reflection by the shear band [20] in these experiments probably due to the weak impedance contrast.

Let us now compare quantitatively the shear waves speed decrease measured during the first shear loading with the prediction by the heuristic EMT model. Using together with a decrease of $Z$ by $10 \%$ at a global shear strain $\varepsilon=U / H \sim 10 \%$ [inset of Fig. 2(b)] and a decrease of a normal stress by $30 \%$ (due to the rotation of the principal stress axis $\sim 45^{\circ}$ and the inhomogeneous repartition of the force network [9]), Eq. (1) predicts a sound speed decrease by $8 \%$. This estimation is in agreement with the decrease of $V_{P}(\sim 10 \%)$, but only half the variation of $V_{S}$ observed in experiments. Two possible reasons might be responsible for such discrepancy. One stems from the heuristic model which does not account for quantitatively either the inhomogeneous field of contact forces, inherent of the direct shear box or the important fabric variation $[8,9]$; note that the shear wave speed $V_{S}$ was shown to be particularly sensitive to the fabric anisotropy [16]. The other, more fundamentally, originates from the breakdown of the EMT in sheared media near unjamming transition (i.e., failure) where the elastic weakening due to relaxation or slippage of grains especially at low confining pressure might induce 

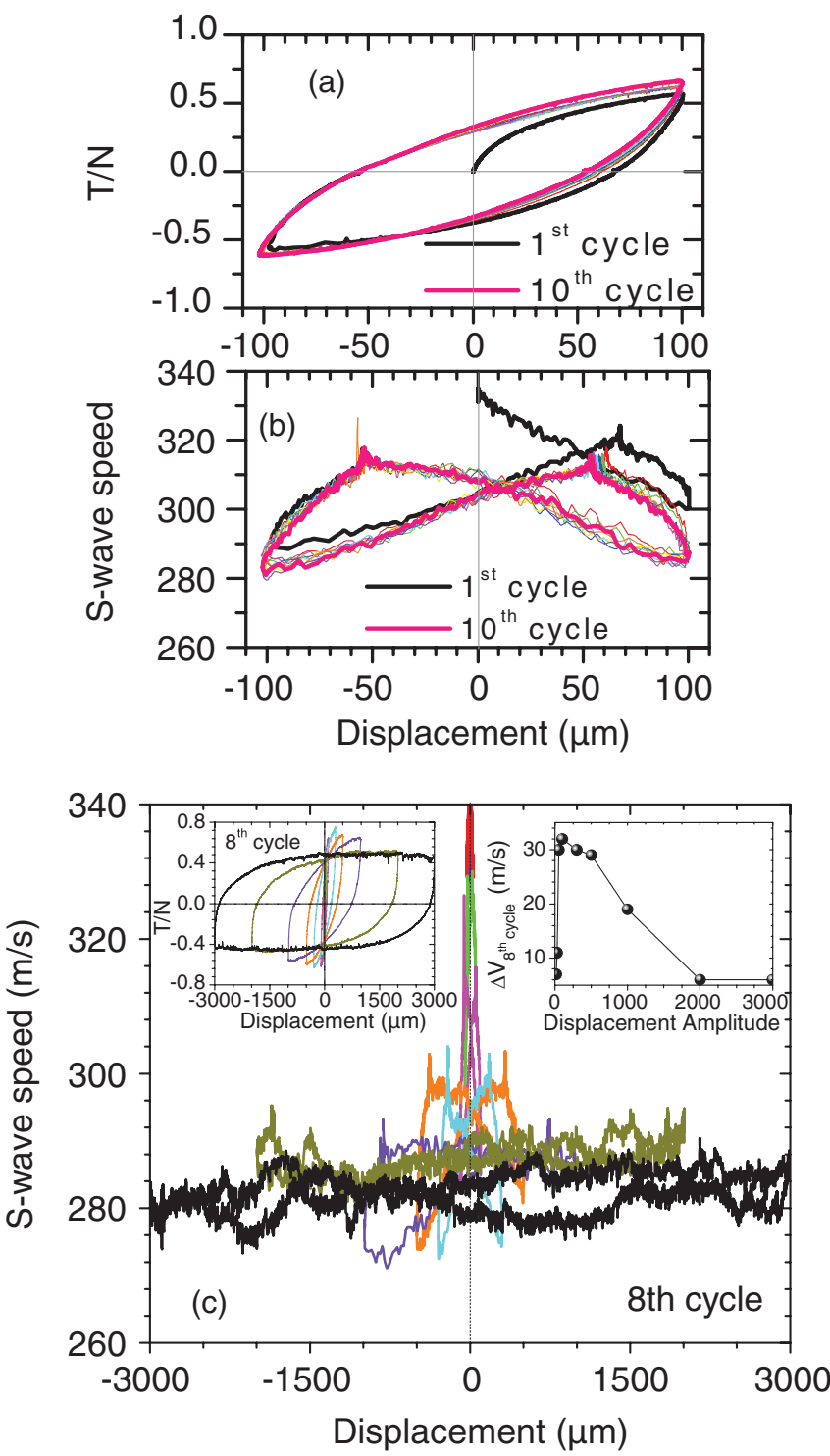

FIG. 3. (Color online) Evolutions of force ratio (a) and shear wave speed (b) under cyclic shear. (c) Evolutions of the shear wave speed during the eighth cyclic loading for different displacement amplitudes. The left inset illustrates the evolution of force ratio during the eighth cycle and the right inset illustrates the evolution of the wave speed variation within the eighth cycle.

a supplementary decrease of $V_{S}$ by $20 \%-40 \%[15,17,21]$. Indeed, if one considers the distinct properties of the shear band formed after the failure (with a height $h=5-10 d$ ) compared to the rest of the medium assumed weakly modified, an increase of $20 \%$ of the time of flight in this stratified medium $t=(H-h) / V_{0}+h / V_{\mathrm{SH}}($ at $\varepsilon=10 \%)$ relative to that under zero shear $t_{0}=H / V_{0}$, might reveal a decrease of shear wave speed $V_{\mathrm{SH}}$ inside the shear band up to $45 \%-70 \%$, not predicted by the EMT model.

\section{A. Cyclic shear}

To better understand the structural changes which lead to the strain localization, we investigate the response of the medium subjected to cyclic shear with increasing shear amplitude.
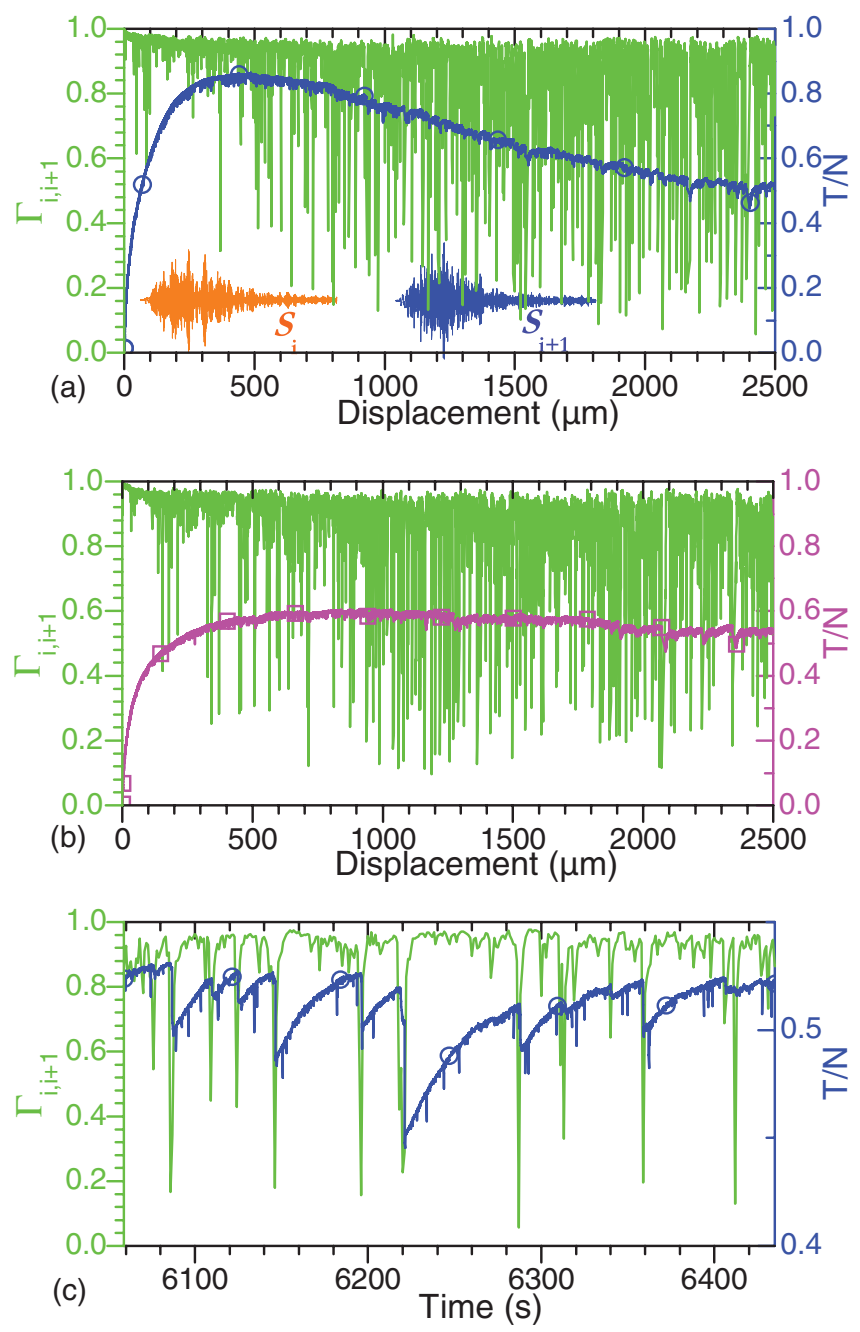

FIG. 4. (Color online) Correlation between two successive coda waves before failure in (a) a dense sample; $\ominus$, force ratio; (b) a loose sample; $\boxminus$, force ratio; and (c) zoomed part of Fig. 4(a) as a function of the experimental time (see text).

In the following, we will focus our attention on the densely packed samples. At a given shear displacement before reaching the peak force, we obtain the typical hysteretic loop between normalized shear force $(T / N)$ and displacement [Fig. 3(a)]. For this small shear displacement, a slight strengthening with the number of cycling is observed. In contrast, for larger amplitude we obtain an elastic weakening (not shown). The evolution of the shear wave speed during this cyclic shear loading is depicted in Fig. 3(b). The remarkable speed decrease between the beginning and the end of the first cycle of shear loading corresponds to some plastic rearrangement and probably small adaptation of the initial packing. Under subsequent unloading-reloading cycles, we observe a repeated hysteretic response of the shear wave speed or elastic modulus as a function of shear displacement; this is likely associated with the evolution of the microstructure when the system explores different metastable configurations via flips events [24]. Such elastic hysteretic behavior is consistent with the evolution of fabric anisotropy obtained in 2D simulation during cyclic tilting of a granular pile below the avalanche angle [25,26]. Indeed, the wave speed varies when the anisotropy and the 
mean coordination number change; the agreement between our wave speed measurements and numerical simulation confirms the ability of sound waves for probing the structural evolution under shear.

Let us now investigate the amplitude effect of the shear displacement. We present in Fig. 3(c) the variations of the shear wave speed during the eighth cyclic loading with different displacement amplitudes [left inset of Fig. 3(c)]. As the amplitude increases, we observe that the maximum variation of the wave speed $\Delta V_{S}$ within the hysteretic loop decreases. As shown in [9], for small displacement, there is no strain localization and the structural change entails the whole packing. Hence, in this regime, the larger the shear displacement, the larger the wave speed variation. Beyond a certain threshold of the shear displacement, the variation of the wave speed reduces importantly due to the onset of the strain localization. In that regime, as was stated above, the packing away from the narrow shear zone does not respond significantly to the shear loading. $V_{S}$ measured from the time of flight across the entire packing varies little and becomes almost constant with the full development of the shear band.

\section{B. Intermittent dynamics before failure}

If the residual strength of a granular medium is independent of the initial state of the granular packing, the peak force and the prepeak behavior such as creep and plastic deformation depend strongly on the packing density and the fabric. Several works have shown that significant precursor events or irreversible rearrangement of grains occur before the peak force, i.e., failure [3-6].

To investigate the precursor behavior, we use the configuration-specific specklelike scattered waves to monitor the granular packings under shear, from the jammed state to the flowing state. More specifically, we define the degree resemblance $\Gamma_{i, j}$ between two successive acoustic speckles $S_{i}(t)$ and $S_{j}(t)$ (inset of Fig. 4) [17]:

$$
\Gamma_{i, j}(\tau=0)=C_{i j}(\tau=0) / \sqrt{C_{i i}(0) C_{j j}(0)}
$$

where $C_{i j}(\tau)$ is the cross-correlation function between $S_{i}(t)$ and $S_{j}(t)$ and $\tau$ is the time lag. Figure 4 illustrates $\Gamma_{i, i+1}$ deduced from two successive scattered acoustic signals recorded at a rate of $1 \mathrm{~Hz}$ in dense and loose bead packings, respectively. This correlation function reveals basically a slight global decorrelation lasting over the experimental range, superposed by the very large pulselike components which occur intermittently. These spikes likely correspond to the major irreversible rearrangements in granular packings evidenced by the intermittent events in the force measurement [Fig. 1(b)]. For closer examination, we plot in Fig. 4(c) a zoomed part of Fig. 4(a) (around $U=2400 \mu \mathrm{m}$ ) as a function of the experimental time. Here the abrupt force drops clearly coincide with the important decorrelation of acoustic speckles $\Gamma_{i, i+1}$. We note that the significant decorrelation may also occur due to the force network change but without visible motions of grains [17]. Furthermore, the intermittent events detected in $\Gamma_{i, i+1}$ appears more pronounced in the loose packing than in the dense packing, before the failure (i.e., peak force). In the following state when the strain is localized, $\Gamma_{i, i+1}$ displays the similar intermittent responses in the two packings, showing that the rheological behavior is henceforth dominated by the shear band.

\section{CONCLUSION}

The evolutions of the structure and the mechanical properties of glass bead packings under shear are investigated by acoustic measurements, during the transition from the jammed state to the flowing state. Measurements of the shear wave speed clearly evidence the breaking of contacts or decrease of the coordination number in sheared granular media; they also provide the acoustic signature when the shear bands are formed. The hysteretic responses under cyclic shear reveal flips or collective rearrangement events. Moreover, the correlation functions of specklelike scattered sound waves evidence the specific intermittent dynamics of the contact force networks. Our results obtained in the laboratory experiments, both for the shear-band formation and the precursor events, may have important implications on large-scale field experiments for remote probing of the fault core dynamics $[4,18]$.

\section{ACKNOWLEDGMENTS}

We thank C. Caroli and T. Baumberger for suggesting the study of shear bands.
[1] D. M. Mueth, G. F. Debregeas, G. S. Karczmar, P. J. Eng, S. R. Nagel, and H. M. Jaeger, Nature (London) 406, 385 (2000).

[2] G. D. R. MiDi, Eur. Phys. J. E 14, 341 (2004).

[3] S. Nasuno, A. Kudrolli, and J. P. Gollub, Phys. Rev. Lett. 79, 949 (1997).

[4] C. Marone, Annu. Rev. Earth Planet Sci. 26, 643 (1998).

[5] L. Staron, J.-P. Vilotte, and F. Radjai, Phys. Rev. Lett. 89, 204302 (2002).

[6] A. Kabla, G. Debrégeas, J.-M. di Meglio, and T. J. Senden, Europhys. Lett. 71, 932 (2005).

[7] K. Iwashita and M. Oda, Powder Technol. 109, 192 (2000).

[8] L. Cui and C. O’Sullivan, Géotechnique 56, 455 (2006).
[9] J. Wang, J. E. Dove, and M. S. Gutierrez, Géotechnique 57, 513 (2007).

[10] T. S. Majmudar and R. P. Behringer, Nature (London) 435, 1079 (2005).

[11] J. Desrues, R. Chambon, M. Mokni, and F. Mazerolle, Géotechnique 46, 529 (1996).

[12] M. Oda and H. Kazama, Géotechnique 48, 465 (1998).

[13] C. H. Liu and S. R. Nagel, Phys. Rev. Lett. 68, 2301 (1992).

[14] X. Jia, C. Caroli, and B. Velicky, Phys. Rev. Lett. 82, 1863 (1999).

[15] H. A. Makse, N. Gland, D. L. Johnson, and L. Schwartz, Phys. Rev. E 70, 061302 (2004). 
[16] Y. Khidas and X. Jia, Phys. Rev. E 81, 021303 (2010).

[17] X. Jia, T. Brunet, and J. Laurent, Phys. Rev. E 84, 020301(R) (2011).

[18] H. J. Melosh, Nature (London) 379, 601 (1996); D. R. Scott, ibid. 381, 592 (1996); P. A. Johnson, H. Savage, M. Knuth, J. Gomberg, and C. Marone, ibid. 451, 57 (2008).

[19] B. Andreotti, Phys. Rev. Lett. 93, 238001 (2004); S. Douady, A. Manning, P. Hersen, H. Elbelrhiti, S. Protiere, A. Daerr, and B. Kabbachi, ibid. 97, 018002 (2006); S. Kiesgen de Richter, V. Y. Zaitsev, P. Richard, R. Delannay, G. Le Caër, and V. Tournat, J. Stat. Mech. (2010) P11023.

[20] C. Caroli and B. Velicky, Phys. Rev. E 67, 061301 (2003).
[21] C. S. O’Hern, L. E. Silbert, A. J. Liu, and S. R. Nagel, Phys. Rev. E 68, 011306 (2003); M. Wyart, S. R. Nagel, and T. A. Written, Europhys. Lett. 72, 486 (2005); M. van Hecke, J. Phys.: Condens. Matter 22, 033101 (2010).

[22] F. Tatsuoka, The 2000 Burmister Lecture (Columbia University, New York, 2000).

[23] P. J. Digby, J. Appl. Mech. 48, 803 (1981); K. Walton, J. Mech. Phys. Solids 35, 213 (1987).

[24] A. Lemaître and C. Caroli, Phys. Rev. Lett. 103, 065501 (2009).

[25] S. Deboeuf, O. Dauchot, L. Staron, A. Mangeney, and J. P. Vilotte, Phys. Rev. E 72, 051305 (2005).

[26] J. Zhang, T. S. Majmudar, A. Tordesillas, and R. P. Behringer, Granular Matter 12, 159 (2010). 